\title{
A New Case of New Daily Persistent Headache Successfully Treated with Botulinum Toxin Type A
}

\author{
Marco Trucco $^{1 *}$, Luigi Ruiz ${ }^{2}$ \\ ${ }^{1}$ Department of Neurology, Headache Centre, Santa Corona Hospital, Pietra Ligure, SV, Italy \\ ${ }^{2}$ Department of Neurology, Headache Centre, SS. Antonio e Biagio e Cesare Arrigo Hospital, Alessandria, Italy \\ Email: *info@marcotrucco.it, lruiz@ospedale.al.it
}

How to cite this paper: Trucco, M. and Ruiz, L. (2019) A New Case of New Daily Persistent Headache Successfully Treated with Botulinum Toxin Type A. Open Access Library Journal, 6: e5786.

https://doi.org/10.4236/oalib.1105786

Received: September 13, 2019

Accepted: October 19, 2019

Published: October 22, 2019

Copyright $\odot 2019$ by author(s) and Open Access Library Inc.

This work is licensed under the Creative Commons Attribution International License (CC BY 4.0).

http://creativecommons.org/licenses/by/4.0/

\begin{abstract}
Objective: Our study describes a typical case of New Daily Persistent Headache $(\mathrm{NDPH})$, refractory to different therapies, that presented a satisfactory response to repeated cycles of botulinum toxin type A. Background: New Daily Persistent Headache is a primary headache disorder, characterized by chronic and unremitting daily headache with abrupt onset and more than three months in duration. It lacks typical clinical features, being similar to chronic migraine without aura or tension-type headache. It may be self-limiting within months or years without therapy, or be refractory to most treatments. Case Report: A 56-year-old woman suffered since eleven years from a daily and continuous headache, pressing/tightening in quality and of moderate intensity, but with daily paroxysms of stabbing pain, without autonomic symptoms. No trigger factors of pain were detected. She experienced a remission period of three years, after that the pain relapsed with the same features. Neurological examination was negative, apart from minimal dysarthria. She was submitted to various examinations (including a complete psychiatric evaluation) and finally diagnosed as NDPH. Her pain was unresponsive to multiple pharmacological treatments. Results: After a week from first cycle of botulinum toxin type A (195 U) she experienced a satisfactory relief of pain and good improvement of her quality of life. Her pain relapsed shortly before subsequent administration of Botox and after a missed cycle: a good relief was subsequently obtained with further cycles of the treatment. Conclusions: This is our second case of NDPH successfully treated with Botulinum Toxin type A. We propose this treatment for refractory cases of chronic daily headaches, including NDPH.
\end{abstract}

\section{Subject Areas}

Neurology 


\section{Keywords}

Headache, Chronic Headache, New Daily Persistent Headache, Botulinum Toxin Type A

\section{Introduction}

New Daily Persistent Headache (NDPH) is a primary headache disorder, characterized by sudden onset (clearly remembered by the patient), followed by chronic and unremitting daily headache within 24 hours, lasting more than three months. It was first described by Vanast [1] in 1986 and later clinically better characterized [2]. It lacks typical clinical features, the pain being suggestive of chronic migraine without aura or tension-type headache. A percentage of NDPH sufferers may report some precipitating events such as a flu-like illness, surgical interventions or stressful events [3]. It may be self-limiting within months or years without therapy, or be refractory to most treatments, frequently representing a major therapeutic challenge.

The pathogenesis of NDPH is unknown, but in some cases association with viral infections and elevation of CSF Tumor Necrosis Factor $\alpha$ (TNF $\alpha$ ) levels, a pro-inflammatory cytokine involved in brain immune and inflammatory processes [4], were reported. A pathogenetic hypothesis is a Central Nervous System (CNS) inflammation, triggered by a viral infection. One paper described a widespread joint hypermobility, specifically in the cervical spine, as a predisposing factor for the development of NDPH [5]. In Table 1 diagnostic criteria from the International Classification of Headache Disorders, $3^{\text {rd }}$ Edition is reported.

We describe a case of NDPH, refractory to different treatments, who after first administration of botulinum toxin type A (195 U) presented a satisfactory remission of pain.

\section{Case Report}

P. I., Female, a 56-year-old woman, first seen in October 2017, overweight (BMI 35.2), referred since August 7, 2006 (this date was specifically remembered) the onset of a daily and continuous headache (without a circadian rhythmicity), localized in the forehead bilaterally, of pressing/tightening quality, with daily paroxysms of stabbing pain of disabling intensity, at the frequency of 1 - 4 per day,

Table 1. ICHD-3 Diagnostic Criteria for NDPH.

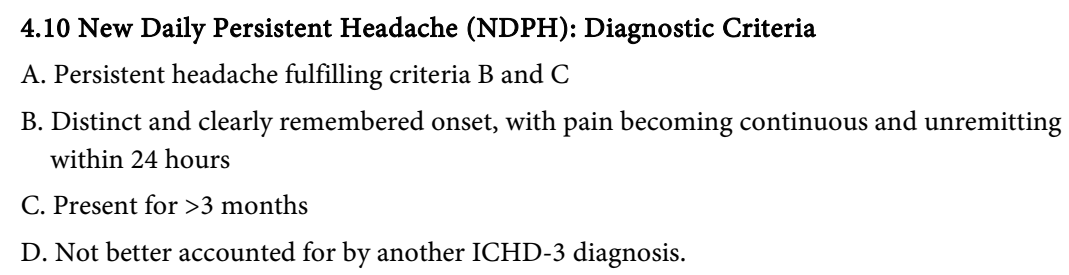


and without general and local autonomic symptoms. The intensity of continuous pain was generally moderate (Visual Analogue Scale 5/10). No headache history, familial headache, head or cervical traumas, surgical interventions, febrile illness prior to the onset of pain, nor psychiatric features were reported. The quality of life (QoL) of the patient was deeply affected by the continuous headache.

The patient complained of frequent lipothymic episodes; during one of these (in 2013) she suffered from a minor cranial injury; after this episode she experienced spontaneous remission of pain for three years. The headache later relapsed without recognizable trigger factors, with the same features.

She underwent various programs for weight reduction, without success.

Neurological examination was unremarkable, apart from minimal dysarthria. No muscular strain or hypertonia were found, not at cervical level nor generalized; no painful trigger points leading to a suspected diagnosis of fibromyalgia were detected.

She underwent different examinations; among these, routine blood tests, screening for thrombophilia, brain Angio-MRI, brain CT scan after head injury, Visual Evoked Potentials, EEG, Eco Doppler of carotid and vertebral arteries and transcranial Doppler (all normal), Tilt Table Test (slight enhancement of vagal tone, no worsening of pain), psychiatric evaluation with Structured Clinical Interview for DSM-IV-TR, Beck Depression Inventory, STAI test and Minnesota Multiphasic Personality Inventory-2 (MMPI-2) (somatic anxiety without mood disorders), evaluation of vegetative control of cardiovascular reflexes (negative) were performed.

The pain was refractory to various symptomatic drugs, including NSAIDs and acetaminophen (at the time of first visit she did not take any symptomatic treatment). As prophylactics, she was treated with anti-depressive drugs as amytriptiline, sertraline, duloxetine, all ineffective; also topiramate, acetylsalicylic acid, and later cycles of oral steroids and verapamil were prescribed without success.

After informed consent, she was treated with botulinum toxin type A (Botox) 195 U s. c. since November 2017, with cycles every three months, following PREEMPT sites of injection and dose paradigm for chronic migraine plus follow-the-pain pattern [6]. The pain relieved about a week after first cycle and relapsed with very mild intensity (VAS 1 - 2) about five-seven days before subsequent administration of Botox (the same also for second and third cycle). After the third cycle she missed one administration (scheduled for August 2018) and complained of a relapse of mild continuous pain, with more severe exacerbations; a new cycle was performed in September 2018, and after this date she experienced intermittent mild headache, with a frequency of every other day, until a new cycle was performed in December 2018, after which the pain subsided almost completely.

The patient is still regularly under treatment, and refers only sporadic mild headache attacks, for which she is not taking medication. 
She does not complain of side effects, aside mild transient injection-site pain and slight local oedema. The patient also reports a substantial improvement of her QoL.

\section{Discussion}

The clinical features of headache, with clearly remembered onset and subsequent continuous pattern (apart from a spontaneous remission after a head trauma), with characteristics of tension-type headache but with stabbing paroxysms, the substantial normality of clinical and instrumental examinations and the refractoriness to most therapies were in accordance with a diagnosis of NDPH (maybe a relapsing-remitting form). A specific trigger factor for the onset of the headache could not be determined.

This is our second case of NDPH treated with botulinum toxin type A. We postulated possible efficacy of the treatment on the basis of some similarities between clinical presentation of NDPH and chronic migraine and of the reported quick responsiveness of this headache entity in a single case, at the dose of 100 units for three cycles of therapy [7], and other unpublished cases.

We confirmed these observations with our previous case report [8]. In the present case we can reasonably exclude a spontaneous remission of the headache because of the relapse of less severe pain after one missed cycle of Botox. Botulinum toxin type A was the only effective treatment and strongly contributed to the improvement of QoL of the patient.

Recent papers [3]-[9], including a single-center retrospective chart review [10], recommended various patterns of this treatment, in particular at PREEMPT dose of $155 \mathrm{U}$, but we used and recommend a higher dose of Botox (195 U), for better efficacy without remarkable side effects. This therapy is still not approved in Italy for NDPH, due to the lack of controlled studies.

The etiology of NDPH and mechanism of action of Botulinum toxin type A in this primary headache disorder are unknown. A state of persistent CNS inflammation secondary to glial activation and increased TNF- $\alpha$ level was postulated as pathogenetic. The efficacy of Botox in NDPH could arise from its central analgesic action due to the inhibition of release of neurotransmitters increasing neurogenic inflammation and central sensitization.

\section{Conclusion}

We believe our cases could represent a further stimulus to perform more observations (i.e. randomized controlled prospective studies) on the efficacy of botulinum toxin type A therapy in chronic refractory primary headaches, including NDPH, in view of the inclusion in national and international therapeutic guidelines.

\section{Consent}

The patient expressed in writing informed consent to the publications of data 
concerning her illness and treatment.

\section{Conflicts of Interest}

The authors declare no conflicts of interest regarding the publication of this paper.

\section{References}

[1] Vanast, W.J. (1986) New Daily Persistent Headache: Definition of a Benign Syndrome. Headache, 26, 317.

[2] Prakash, S., Saini, S., Rana, K.R. and Mahato, P. (2012) Refining Clinical Features and Therapeutic Options on New Daily Persistent Headache: A Retrospective Study of 63 Patients in India. The Journal of Headache and Pain, 13, 477-475.

https://doi.org/10.1007/s10194-012-0461-6

[3] Rozen, T.D. (2014) New Daily Persistent Headache: An Update. Current Pain and Headache Reports, 18, 431. https://doi.org/10.1007/s11916-014-0431-6

[4] Rozen, T. and Swidan, S.Z. (2007) Elevation of CSF Tumor Necrosis Factor $\alpha$ (TNF $\alpha$ ) Levels in New Daily Persistent Headache and Treatment Refractory Chronic Migraine. Headache, 47, 1050-1055.

https://doi.org/10.1111/j.1526-4610.2006.00722.x

[5] Rozen, T.D., Roth, J.M. and Denenberg, N. (2006) Cervical Spine Joint Hypermobility: A Possible Predisposing Factor for New Daily Persistent Headache. Cephalalgia, 26, 1182-1185. https://doi.org/10.1111/j.1468-2982.2006.01187.x

[6] Blumenfeld, A., Silberstein, S.D., Dodick, D.W., Aurora, S.K., Turkel, C.C. and Binder, W.J. (2010) Method of Injection of onabotulinumtoxinA for Chronic Migraine: A Safe, Well-Tolerated, and Effective Treatment Paradigm Based on the PREEMPT Clinical Program. Headache, 50, 1406-1418.

https://doi.org/10.1111/j.1526-4610.2010.01766.x

[7] Spears, R.C. (2008) Efficacy of Botulinum Toxin Type A in New Daily Persistent Headache. The Journal of Headache and Pain, 9, 405-406. https://doi.org/10.1007/s10194-008-0078-y

[8] Trucco, M. and Ruiz, L. (2018) New Daily Persistent Headache Treated with Botulinum Toxin Type A: Case Report. Austin Journal of Anatomy, 5, 1082.

[9] Nierenburg, H. and Newman, L.C. (2016) Update on New Daily Persistent Headache. Current Treatment Options in Neurology, 18, 25. https://doi.org/10.1007/s11940-016-0408-3

[10] Ali, A., Kriegler, J., Tepper, S. and Vij, B. (2019) New Daily Persistent Headache and OnabotulinumtoxinA Therapy. Clinical Neuropharmacology, 42, 1-3.

https://doi.org/10.1097/WNF.0000000000000313 